Provided for non-commercial research and education use. Not for reproduction, distribution or commercial use.

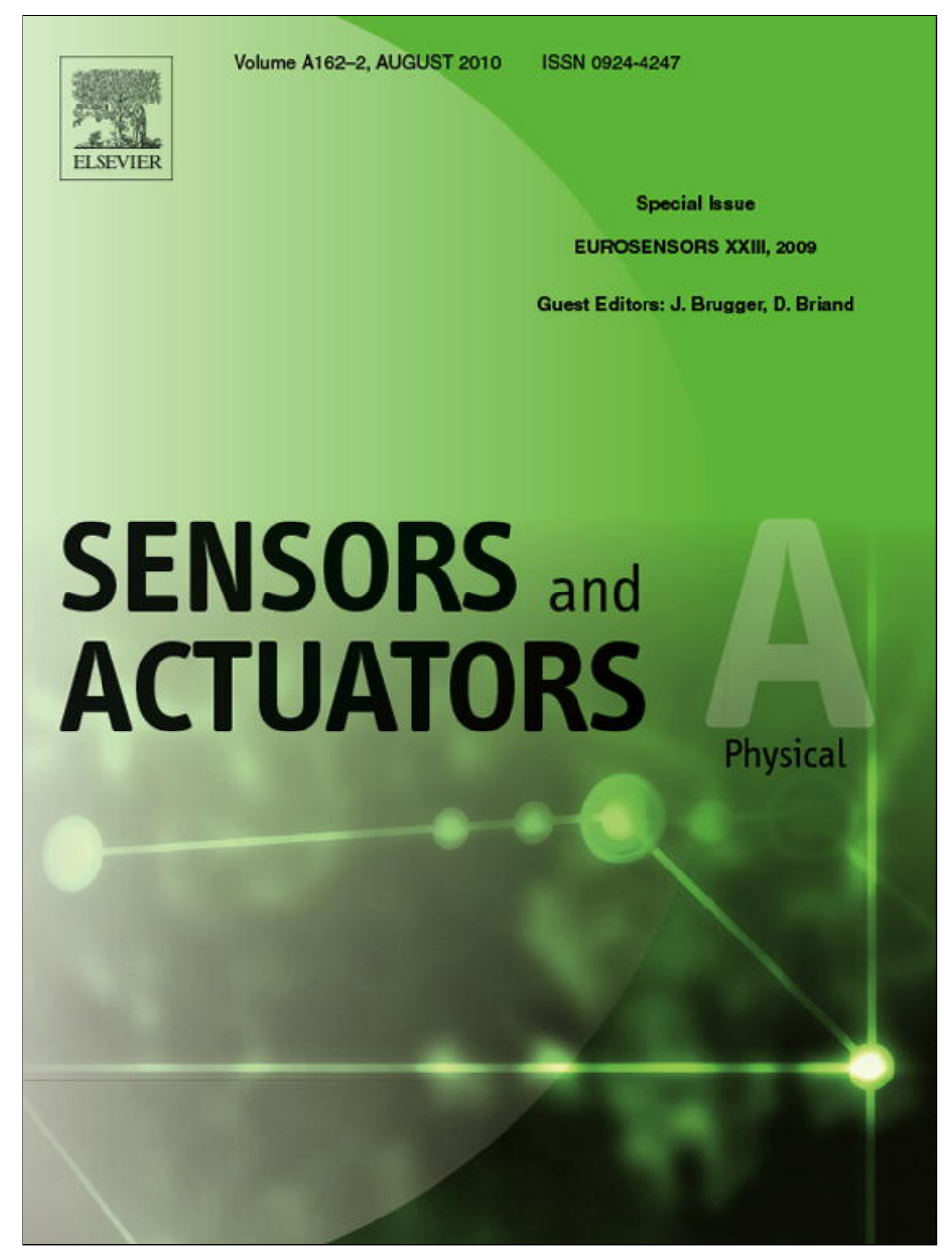

This article appeared in a journal published by Elsevier. The attached copy is furnished to the author for internal non-commercial research and education use, including for instruction at the authors institution and sharing with colleagues.

Other uses, including reproduction and distribution, or selling or licensing copies, or posting to personal, institutional or third party websites are prohibited.

In most cases authors are permitted to post their version of the article (e.g. in Word or Tex form) to their personal website or institutional repository. Authors requiring further information regarding Elsevier's archiving and manuscript policies are encouraged to visit:

http://www.elsevier.com/copyright 


\title{
Improved energy harvesting from wideband vibrations by nonlinear piezoelectric converters
}

\author{
M. Ferrari ${ }^{\mathrm{a}, *}$, V. Ferrari $^{\mathrm{a}}$, M. Guizzetti ${ }^{\mathrm{a}}$, B. Andò ${ }^{\mathrm{b}}$, S. Baglio $^{\mathrm{b}}$, C. Trigona $^{\mathrm{b}}$ \\ a Dipartimento di Ingegneria dell'Informazione, University of Brescia, Via Branze 38, 25123 Brescia, Italy \\ ${ }^{\mathrm{b}}$ Dipartimento di Ingegneria Elettrica Elettronica e dei Sistemi, University of Catania, Viale Andrea Doria 6, I-95125 Catania, Italy
}

\section{A R T I C L E I N F O}

\section{Article history:}

Received 4 November 2009

Received in revised form 12 May 2010

Accepted 15 May 2010

Available online 9 June 2010

\section{Keywords:}

Energy harvesting

Piezoelectric converter

Wide-spectrum vibrations

Nonlinear bistable system

Stochastic resonance

\begin{abstract}
A B S T R A C T
Vibration harvesters typically are linear mass-spring devices working at resonance. A different approach is here proposed based on nonlinear converters that exploit stochastic resonance with white-noise excitation. It consists of a piezoelectric beam converter coupled to permanent magnets to create a bistable system. Under proper conditions, the system bounces between two stable states in response to random excitation, which significantly improves energy harvesting from wide-spectrum vibrations. The background theory is discussed based on a simplified monodimensional model which includes nonlinearity. A cantilever beam with added nonlinearity was simulated by using a MATLAB ${ }^{\circledR}$ Stochastic Differential Equation (SDE) Toolbox demonstrating the expected improvement under white-noise vibrations. Nonlinear converters were then realized by screen printing low-curing-temperature lead zirconate titanate (PZT) films on steel cantilevers equipped with magnets. Experimental tests were performed by measuring both the beam deflection and the output voltage under excitation by random vibrations at varying degree of nonlinearity added to the system. The obtained results show that the performances of the converter in terms of output voltage at parity of mechanical excitation are markedly improved when the system is made bistable. Furthermore, the principle was also preliminarily validated on a MEMS U-shaped cantilever beam that was purposely designed and fabricated in SOI technology. This demonstrates the possibility to downscale the principle here proposed in the perspective of a MEMS harvester based on nonlinear piezoelectric converters.
\end{abstract}

(C) 2010 Elsevier B.V. All rights reserved.

\section{Introduction}

The topic of power harvesting is increasingly considered a key point in the development of autonomous sensing nodes and microsystems with extended lifetimes in unattended operation [1-4]. Among the power harvesting methods, the conversion of mechanical energy from background vibrations into electrical energy via the piezoelectric effect is particularly effective, due to the ubiquitous presence of vibrations and the availability of piezoelectric materials of adequate performances [5-12]. In energy harvesting from vibrations, mechano-electrical converters are typically realized as linear mechanical resonators. The effectiveness of such converters is maximum when operated at resonance, but it is considerably suboptimal with frequency-varying and wideband vibrations. In order to increase the bandwidth of the converter the simplest solution is to decrease the mechanical quality factor $Q$, but this worsens the peak response and the conversion effectiveness. To overcome this limitation more complex methods can be adopted,

\footnotetext{
* Corresponding author. Tel.: +39030 3715899; fax: +39030 380014.

E-mail address: marco.ferrari@ing.unibs.it (M. Ferrari).
}

among which the use of array of generators with different resonance frequencies and nonlinear or bistable structures have been recently reported as particularly effective [13]. The first strategy consists of the use of multiple converters with different frequency responses combined into arrays [14-17]. This allows to increase the equivalent bandwidth without negatively affecting the $Q$-factor of a single converter, thus not decreasing the peak response. However, for a given excitation frequency only one single converter at a time contributes to the power generation, with a corresponding limitation in the whole generated power density. Nonlinearity can be used for example to tune the system linear resonance near the equilibrium point in order to allow less accurate fabrication process, as done by Mann and Sims [18]. The authors realized an electromagnetic harvester that exploits magnetic force to levitate an oscillating magnet with tunable resonance frequency. Beside the capability to tune the resonance frequency, also the possibility to improve the effectiveness of the energy harvester by means of nonlinearity was investigated. The energy converter was modelled and examined in the case of harmonic excitation. This analysis, confirmed by experimental tests, shows that the nonlinear system generates large oscillations over a wider frequency range, with respect to the linear case, thus potentially improving the energy harvested under 
proper conditions. The effect on the amplification of the vibrations of an oscillating energy converter by means of nonlinear forces was also investigated by Shahruz [19]. The proposed harvester is made by a piezoelectric beam with a magnetic proof mass on the tip and magnets in its vicinity. A method to properly set the strength and the position of the magnets in order to achieve the desired nonlinear force was developed. Lin et al. [20] fabricated a piezoelectric converter where a restoring nonlinear force was introduced with permanent magnets. The harvester was harmonically excited while measuring the rectified voltage fed to a $22 \mu \mathrm{F}$ capacitor with a $1 \mathrm{M} \Omega$ resistor in parallel. It was demonstrated that using the nonlinear device there is an increase in the off-resonance output voltage thus enhancing the power generated over a wider frequency spectrum.

Particular types of nonlinear systems are the bistable systems, where two equilibrium positions are present. Bistability can be implemented with a specific design of the mechanical structure of the system [21] or by introducing external nonlinear forces. The presence of bistability makes the system capable to rapidly switch between the stable states under proper mechanical excitations, thereby increasing the velocity and, in turn, the power converted by the harvester, as analytically studied in $[22,23]$. The bistable snap-through mechanism studied and simulated by Ramlan et al. [22] shows an enhancement in the generated power, for this specific system, when the excitation is at frequencies lower than the linear resonance, due to the intrinsic square-wave displacement response of the bistable system. Better performances of bistable converters respect to linear converters in terms of wider spectral response were demonstrated by Gammaitoni et al. [24,25] in case of wide-spectrum vibrations. The authors realized a bistable inverted pendulum that converts mechanical energy by means of piezoelectric effect. The power dissipated on a purely resistive load was computed measuring the rms voltage on it when the converter was excited with random vibrations with a Gaussian distribution. As a general feature of nonlinear systems, the generated power is influenced by both the spectral distribution and the intensity of the input vibrations. In fact, when the vibration magnitude is not high enough, the converter cannot jump between the two stable states but it oscillates around the equilibrium position with a locally linear behaviour.

In this context, the present work proposes and experimentally validates an approach based on a bistable nonlinear oscillator where, by means of magnets, bistability is intentionally introduced in a 2nd order mechanical system represented by a piezoelectric cantilever converter, innovatively realized by screen-printing technology. An analytical model of the nonlinear system has been derived and supported with simulations, to investigate the behaviour and the spectral response of the bistable converter. Both macroscale and microscale nonlinear devices have been considered to validate the scalability of the proposed principle. Under wideband mechanical vibrations, the performances of the bistable converter in terms of output voltage have been measured and compared with the linear case, at parity of excitation. In these conditions, the nonlinear bistable converter has been demonstrated to provide a higher output voltage over a wider frequency spectrum with respect to the linear case.

\section{Theory of operation}

\subsection{Analysis of the principle}

The principle of the proposed converter can be illustrated with reference to a monodimensional undamped nonlinear system formed by a mass $m$ and a spring with stiffness $k$, with the addition of an effect produced by a force $F_{\mathrm{NL}}$ that symmetrically pushes the mass away from equilibrium with an intensity that is a nonlinear

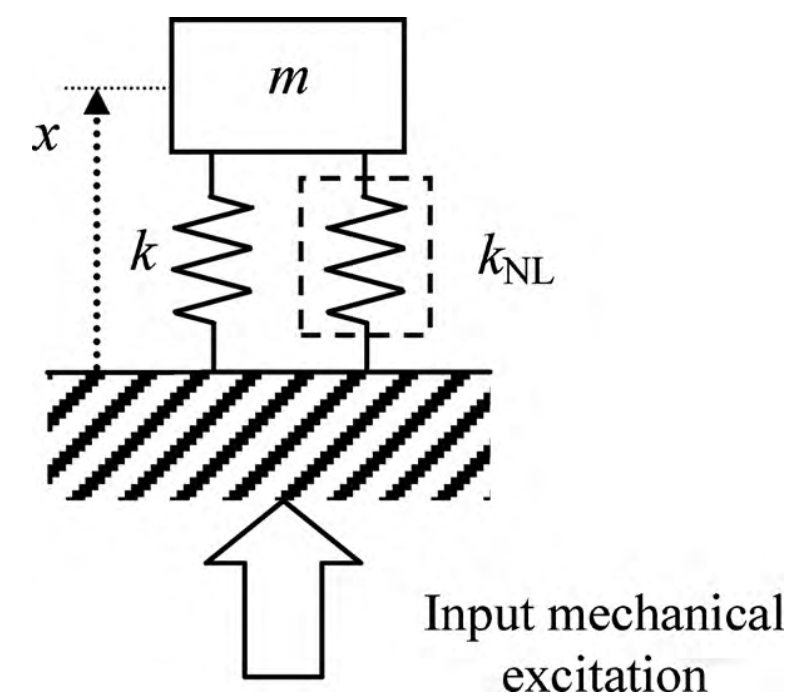

Fig. 1. Equivalent mechanical model for a nonlinear undamped system. Nonlinearity is introduced by an additional nonlinear spring with mechanical stiffness $k_{\mathrm{NL}}$. The term $F_{\mathrm{NL}}$ is the associated nonlinear force.

function of displacement $x$. Fig. 1 shows a mechanical model of the system where the nonlinear effect due to $F_{\mathrm{NL}}$ is represented by the element with nonlinear equivalent stiffness $k_{\mathrm{NL}}$.

When the mass $m$ is displaced from the equilibrium point, a restoring force $F$ due to the linear spring and a counter-restoring force $F_{\mathrm{NL}}$ due to the nonlinear element act on the mass given by:

$F=-k x$

$F_{\mathrm{NL}}=k_{\mathrm{NL}} x$

Due to the assumed symmetry, the force $F_{\mathrm{NL}}$ has to be a nonlinear odd function of $x$, where the simplest function with these features is a 3rd order polynomial given by:

$F_{\mathrm{NL}}=\alpha x-\beta x^{3}$

where the coefficients $\alpha$ and $\beta$ are positive real numbers which depend on the characteristics of the system.

The nonlinear equivalent stiffness $k_{\mathrm{NL}}$ results a nonlinear even function of $x$, given by:

$k_{\mathrm{NL}}=\alpha-\beta x^{2}$

The free movement of the mass given by the displacement $x$ is therefore governed by the following differential equation:

$m \ddot{x}-F_{\mathrm{TOT}}=m \ddot{x}-\left(F+F_{\mathrm{NL}}\right)=m \ddot{x}+\left(k-k_{\mathrm{NL}}\right) x=0$

The potential energy $U(x)$ is obtained by spatial integration of the total force $F_{\text {TOT }}$ acting on the mass $m$, leading to the following expression:

$U(x)=-\int F_{\mathrm{TOT}} d x=-\int\left(F+F_{\mathrm{NL}}\right) d x=\frac{1}{2}(k-\alpha) x^{2}+\frac{\beta}{4} x^{4}$

Fig. 2 plots the potential energy $U(x)$ for different values of $(k-\alpha)$ and $\beta \geq 0$. For $\beta=0$ the system is linear. For $\beta>0$ and $(k-\alpha) \geq 0$, the potential $U(x)$ has only one stable equilibrium point in the origin. In this condition $(k-\alpha)$ represents the equivalent stiffness of the system linearized around the origin, that decreases when $\alpha$ increases. When $\beta>0$ and $(k-\alpha)<0, U(x)$ shows a symmetric double well with two stable equilibrium points and an unstable equilibrium point in the origin, implying a bistable behaviour.

In the latter case, under an external applied force representing the vibrations to be converted, the mass can oscillate near the stable equilibrium points in each well, or it can jump from one well to the other if the supplied mechanical energy is high enough. This 


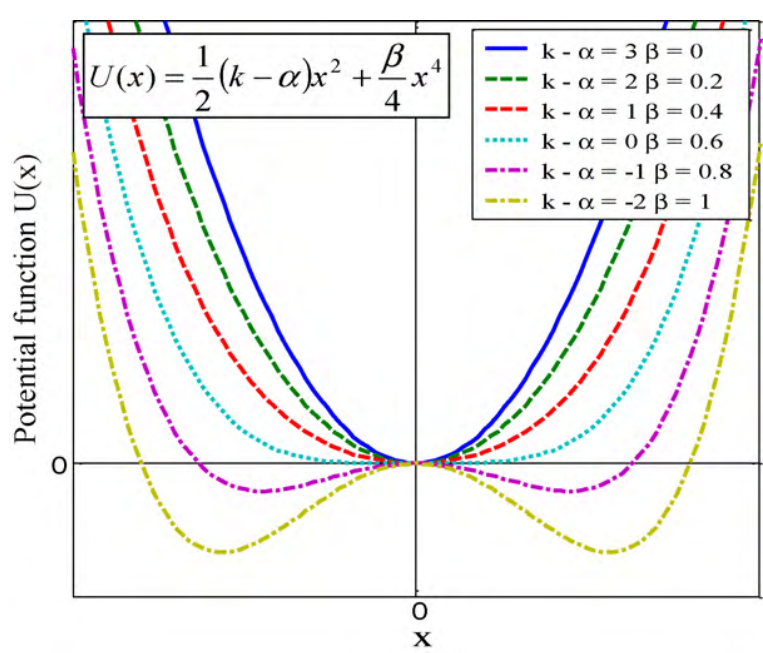

Fig. 2. Potential function $U(x)$ for different values of $(k-\alpha)$ and $\beta$.

produces sudden jumps in the displacement which will ultimately determine an average increase in the converter output.

Considering a cantilever beam, it is possible to create the above described bistability by positioning two permanent magnets with opposite polarities, respectively on the cantilever tip and on an external fixed support at a distance $d$ along the beam axis, as shown in Fig. 3. A repulsive force $F_{R}$ acts between the two magnets, and it decreases in magnitude with increasing the distance $d$.

To perform an approximate analysis, the system can be simplified to the lumped-element monodimensional model of Fig. 4. The mass $m$ accounts for the effective mass of the 1st flexural mode of the cantilever plus the magnet, and the stiffness $k$ represents the elastic reaction. The actual bending of the cantilever tip in Fig. 3 is represented by the vertical displacement $x$ of the mass in the simplified model of Fig. 4.

For a given value of $d$, when the mass moves then the repulsive force $F_{\mathrm{R}}$ changes in direction by an angle $\theta$, but it will be assumed to remain constant in magnitude. This is a further simplifying assumption that for the present purposes is entirely reasonable for small angles $\theta$.

The horizontal component of the force $F_{\mathrm{R}}$ is balanced by the longitudinal stiffness of the cantilever which is assumed sufficiently high. The vertical component $F_{\mathrm{Rv}}$ of $F_{\mathrm{R}}$ affects the motion and it is given by:

$F_{\mathrm{Rv}}=F_{\mathrm{R}} \sin \theta$

The vertical tip displacement $x$ and the angle $\theta$ are related by $x=d \tan \theta$. Therefore, the resulting relationship between the vertical

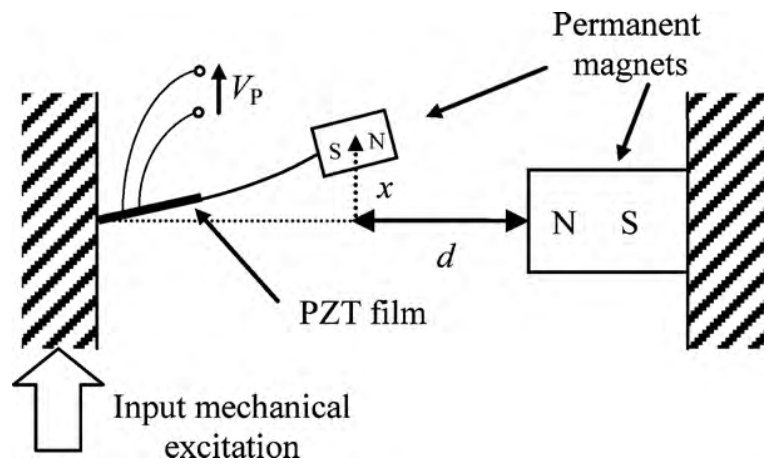

Fig. 3. Bistable system formed by a piezoelectric cantilever beam and two permanent magnets. When distance $d$ is low enough, $(k-\alpha)$ becomes negative and the cantilever bounces between two stable states.

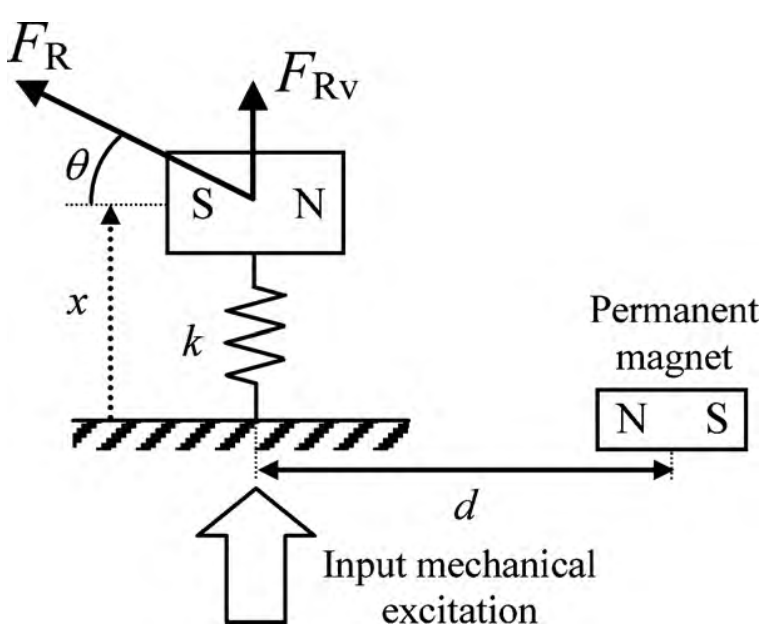

Fig. 4. Geometry of the nonlinear system formed by a piezoelectric cantilever beam and two permanent magnets.

component $F_{\mathrm{Rv}}$ of the repulsive force and $x$ is the following:

$F_{\mathrm{Rv}}=F_{\mathrm{R}} \sin \theta=F_{\mathrm{R}} \frac{\tan \theta}{\sqrt{1+\tan ^{2} \theta}}=\frac{F_{\mathrm{R}}}{d} \frac{x}{\sqrt{1+\left(x^{2} / d^{2}\right)}}$

The vertical force $F_{\mathrm{Rv}}$, as written in (7), can be expanded into its Taylor series computed around $x=0$ stopped at the 3 rd term, obtaining the following expression:

$F_{\mathrm{Rv}} \cong \frac{F_{\mathrm{R}}}{d} x-\frac{F_{\mathrm{R}}}{2 d^{3}} x^{3}$

It can be observed that $F_{\mathrm{Rv}}$ in (8) has the same general form of $F_{\mathrm{NL}}$ in (2) being a nonlinear odd function of the tip displacement $x$. Considering that $F_{\mathrm{R}}$ is actually a decreasing function $F_{\mathrm{R}}(d)$ of the distance $d$, the coefficients $\alpha$ and $\beta$ are given by $F_{R}(d) / d$ and $F_{R}(d) /\left(2 d^{3}\right)$, respectively and both result to be decreasing functions of the distance $d$. In this way, varying the distance $d$ implies a change in the shape of the potential energy of the system, as shown in Fig. 2, thereby creating a monostable or bistable system behaviour. When the system has a bistable behaviour, the beam has two deflected positions of equilibrium, with the possibility to jump between the two states when it is mechanically excitated at the base.

\subsection{Numerical simulations}

Numerical simulations were run on a sample system including a cantilever and the nonlinear effect due to the magnetic force as in Fig. 2. The nonlinear switching mechanism model was expressed as two 1st order Itô's equations [26] and simulated by using MATLAB ${ }^{\circledR}$ SDE (Stochastic Differential Equation) Toolbox [27]. The Eulero-Maruyama method was used and a white-noise vibration source was applied at the cantilever base. Fig. 5 shows the qualitative deflection of the cantilever beam in presence of the bistability and in the linear case, corresponding to finite and exceedingly large distance $d$ between the cantilever and the external magnet, respectively.

Fig. 6 reports the responses in the frequency domain, represented as the velocity power spectral density, normalized to the maximum value. The nonlinear bistable case provides a spectrum with a wider bandwidth compared to resonant behaviour of the linear case. This is expected to produce an improved effectiveness in converting wide-spectrum vibrations. 


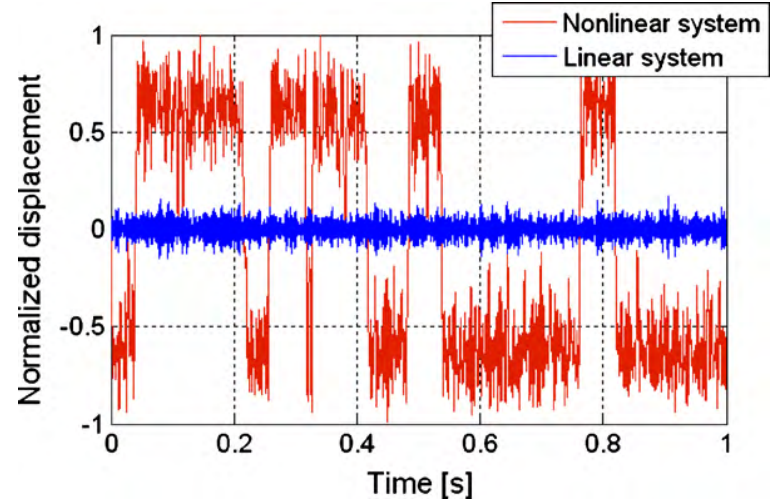

Fig. 5. Simulated deflection of the cantilever in the time domain. The signals are normalized to the maximum value of amplitude. The nonlinear evolution represents the stochastic resonance condition.

\section{Experimental results}

\subsection{Principle validation}

The principle of the nonlinear behaviour was evaluated both at the macroscale and at the microscale. For validation at the macroscale, different sets of piezoelectric bimorph converters were realized by screen printing low-curing-temperature lead zirconate titanate (PZT) films on steel cantilevers [28]. A first set of cantilevers was initially used for the principle validation by focusing on the mechanical behaviour of the devices and performing measurements on the tip displacement. The cantilevers have dimensions of $(40 \mathrm{~mm} \times 5 \mathrm{~mm} \times 0.5 \mathrm{~mm})$. A permanent magnet was fixed on the cantilever tip while an external permanent magnet was fixed on a micrometric stage so that the distance $d$ between the magnets could be varied. The magnets were mounted with opposed polarities, so that the resulting magnetic force was repulsive. A custom designed optical triangulator, realized with the reflective sensor OPB705, was used to measure the cantilever tip displacement, with the setup shown in Fig. 7. With this setup, decreasing the distance $d$ implies an increase of the $\alpha$ and $\beta$ values in Eqs. (2) and (8). The converter was excited at the base by vertical mechanical vibrations generated by an electrodynamic shaker. The shaker was driven by a band-pass filtered white-noise voltage whose normalized amplitude spectrum is shown in Fig. 8.

Fig. 9 shows the measured tip displacement of the cantilever under an applied excitation from the shaker resulting in an rms acceleration $a_{\mathrm{rms}}=0.3 \mathrm{~g}$. As shown in the time records of the measured signals, when the distance $d$ is decreased from large values the system initially maintains a monostable quasi linear behaviour with a progressive decrease in the linearized resonance frequency, i.e. the resonance frequency of the linearized system, until the equivalent stiffness $(k-\alpha)$ is positive. At the same time,

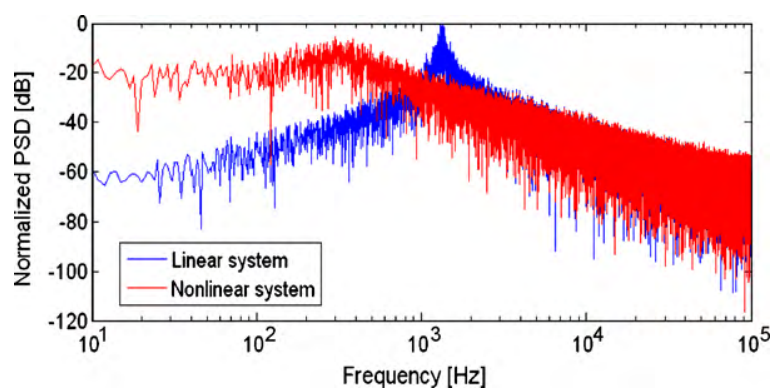

Fig. 6. Simulated velocity power spectral density (PSD) of the cantilever in the frequency domain. The signals are normalized to the maximum value.

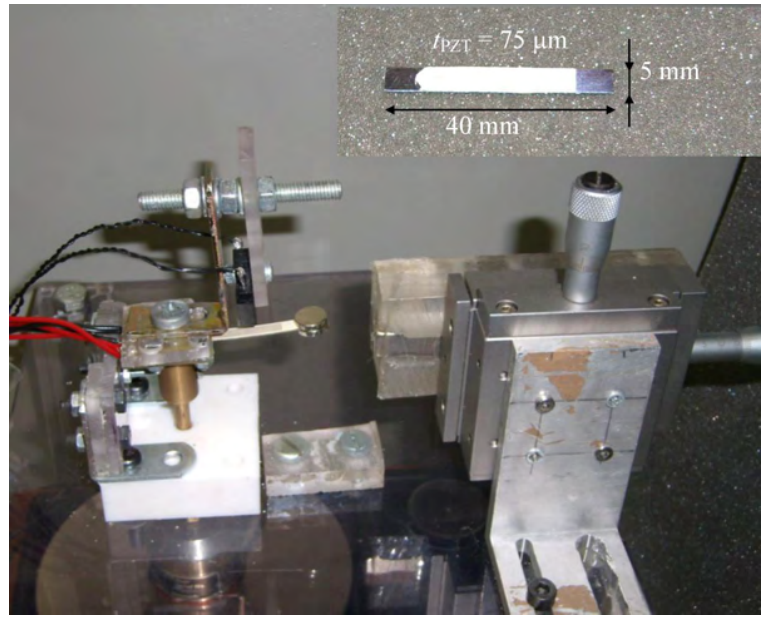

Fig. 7. Experimental setup for bistable system characterization.

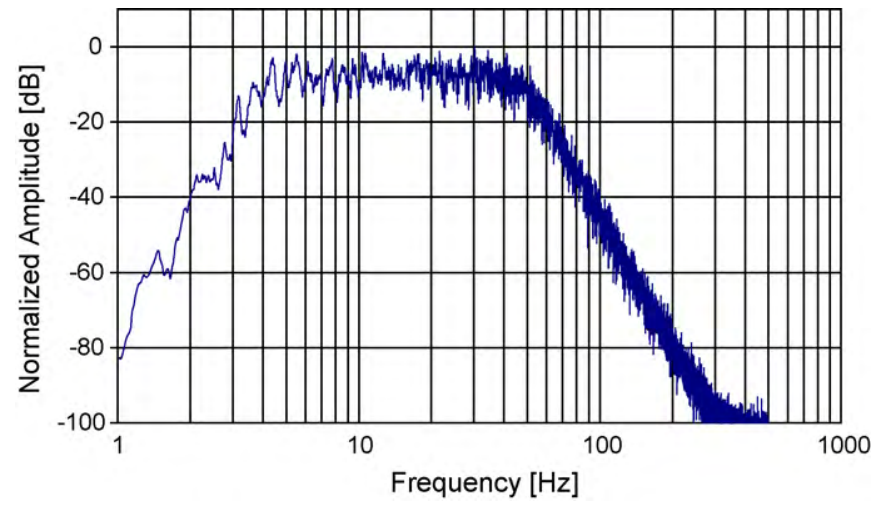

Fig. 8. Frequency spectrum of the input voltage of the electrodynamic shaker used for the excitation of the converter.

the displacement increases due to the decreased equivalent stiffness $(k-\alpha)$. When the magnets are adequately close (bottom plot, $d=2.5 \mathrm{~mm}$ ), the equivalent stiffness $(k-\alpha)$ becomes negative, thereby making the system bistable. In this condition, switching between the two stable states occurs and the fluctuations of the tip displacement significantly increase.

In order to evaluate the proposed approach at the microscale, a MEMS device was designed adopting a custom technology based on a SOI (Silicon On Insulator) substrate, performed at the Centro Nacional de Microelectronica (CNM) in Barcelona, Spain. The designed micromachined structure is a U-shaped cantilever device

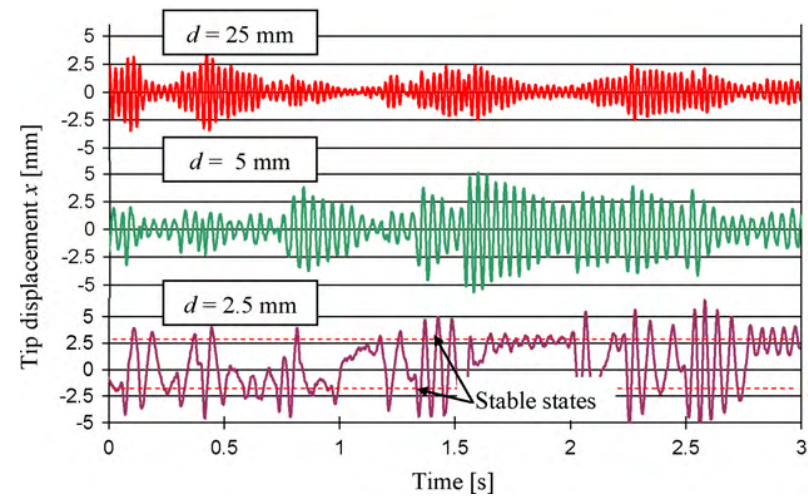

Fig. 9. Tip displacement of the converter for different values of the distance $d$ between the two magnets, with an acceleration of the input vibrations $a_{\mathrm{rms}}=0.3 \mathrm{~g}$. 


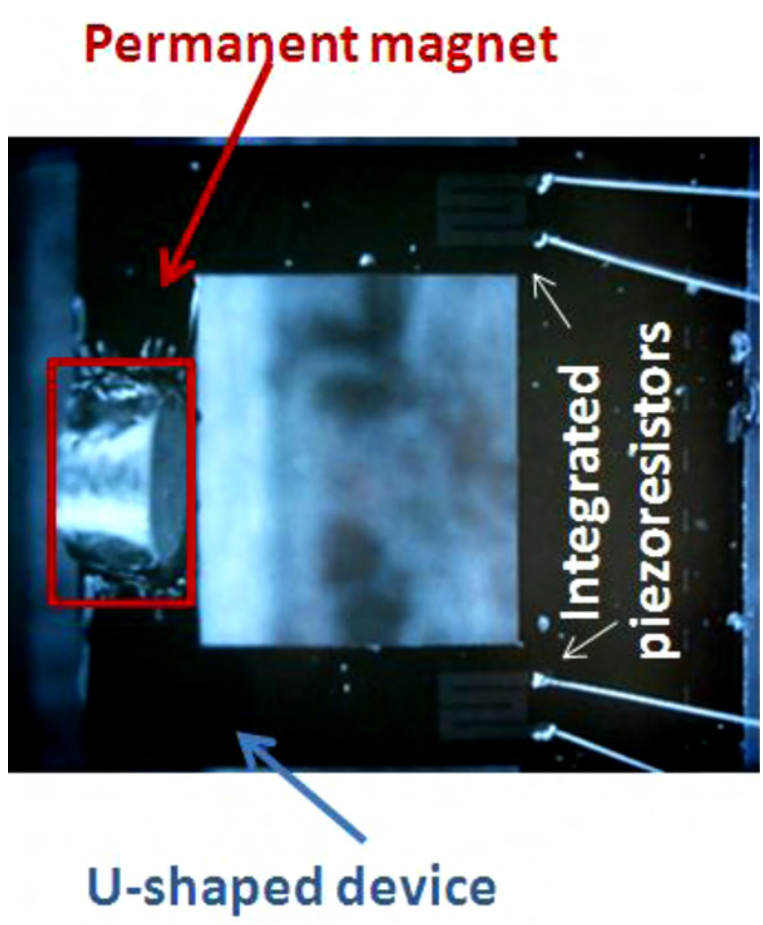

Fig. 10. Microscope picture of the MEMS U-shaped cantilever.

with two parallel clamped beams having length of $2500 \mu \mathrm{m}$ and width of $700 \mu \mathrm{m}$, connected at their free extremity by a 'linking arm' of $3400 \mu \mathrm{m} \times 700 \mu \mathrm{m}$. The thickness of the device is $15 \mu \mathrm{m}$. At the clamped end of each lateral beam, polysilicon piezoresistors were placed to detect the microstructure deflection.

To experimentally evaluate the proposed approach, a permanent magnet was glued on the tip of the U-shaped cantilever as shown in Fig. 10. An external permanent magnet stack was placed in front of the microdevice on a translator system in order to modify the distance $d$ between the two magnets.

A shaker was used to mechanically excite the microstructure. The piezoresistors were connected in a Wheatstone bridge leading to a voltage output, and a LabVIEW ${ }^{\mathrm{TM}}$ routine was developed to drive the shaker using a signal generator (Agilent 33220A) programmed via a GPIB interface. The system dynamics was analyzed using Gaussian white noise with an upper bandwidth limit of $500 \mathrm{~Hz}$ imposed through the shaker, resulting in an applied rms acceleration $a_{\mathrm{rms}}=0.9 \mathrm{~g}$.

Fig. 11 shows a typical time record of the amplified output signal from the bridge when the magnets are suitably close, i.e. in the order of $1 \mathrm{~mm}$ apart, to induce bistability in the system. The results are consistent with theoretical predictions, confirming that the cantilever bounces between the two stable states provided that the excitation is sufficient.

\subsection{Piezoelectric nonlinear converter}

A second set of piezoelectric converters, having the same dimensions of the first set, was used to verify and measure the performances of nonlinear converters in terms of open-circuit output voltage, in comparison with the linear case. The same setup shown in Fig. 7 was used. The system was excited by means of the electrodynamic shaker with mechanical vibrations produced by the same spectrum of Fig. 8 used in the previous experiment on the measurement of tip displacement. In this case, the open-circuit output voltage $V_{\mathrm{P}}$ generated by the piezoelectric converter was measured together with the displacement $x$, at different values of the distance $d$ between the magnets.

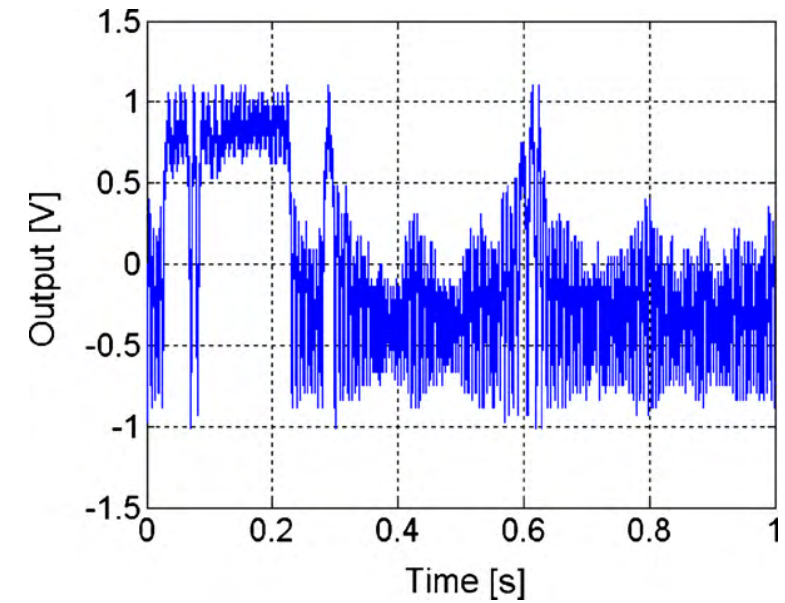

Fig. 11. Output signal of the conditioning circuit (Wheatstone bridge) for $d \sim 0.94 \mathrm{~mm}$.

Fig. 12 shows the typical results obtained for $d=2.5 \mathrm{~mm}$ which was a suitable value to make the system bistable. The measured trend of the tip displacement is in agreement with the results of Fig. 9, and it shows the same bistable behaviour of the simulation results of Fig. 5 (nonlinear system).

Regarding the measured voltage, since the piezoelectric converter can be conveniently modelled by a charge source $Q_{p}$, whose magnitude is proportional to the stress and thus to the tip deflection $x$, in parallel with a capacitance $C_{\mathrm{P}}$ and a leakage resistance $R_{\mathrm{P}}$, the open-circuit output voltage $V_{\mathrm{P}}$ results in a high-pass function of the tip displacement $x$ [29].

This is consistent with the results of Fig. 12 where it can be seen that when a switching in the displacement $x$ occurs, a voltage pulse is generated in $V_{\mathrm{P}}$. As a result, the nonlinear behaviour produces an increase in the open-circuit voltage from the converter, as demonstrated by the computed rms voltage values at different distances $d$ that result $V_{d=25.0 \mathrm{~mm}}=4.27 \mathrm{~V}, V_{d}=5.0 \mathrm{~mm}=6.11 \mathrm{~V}$, and $V_{d=2.5 \mathrm{~mm}}=7.99 \mathrm{~V}$, respectively.

Also the frequency spectra of the open-circuit voltage generated by the piezoelectric converter were measured, as shown in Fig. 13. It can be observed that decreasing the distance $d$ the system initially remains monostable and quasi linear, and at the same time the linearized resonance frequency decreases due to the increase in the equivalent compliance. When $d$ is small enough, i.e. lower than a threshold that was estimated to be around $4 \mathrm{~mm}$, the equivalent stiffness becomes negative and the system in this excitation condition toggles into bistable behaviour, with a change in the shape

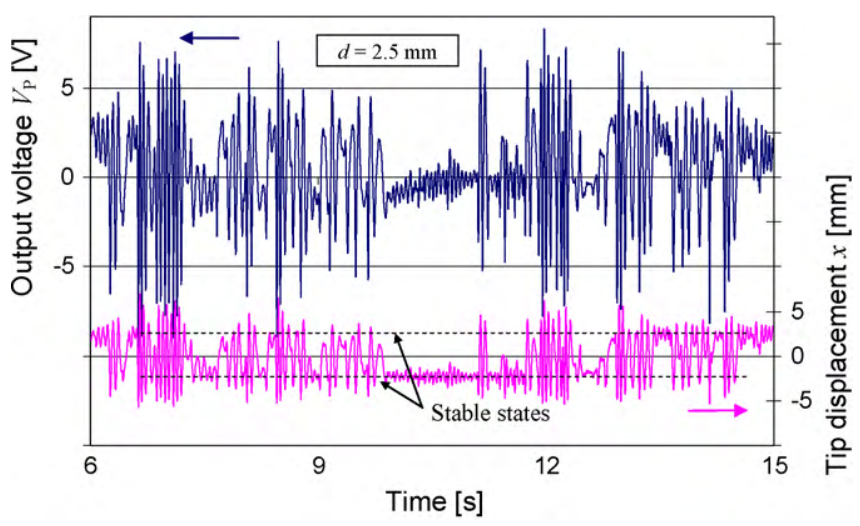

Fig. 12. Output voltage $V_{\mathrm{P}}$ and tip displacement of the piezoelectric converter in a nonlinear behaviour condition, with an acceleration of the input vibrations $a_{\mathrm{rms}}=0.3 \mathrm{~g}$. 


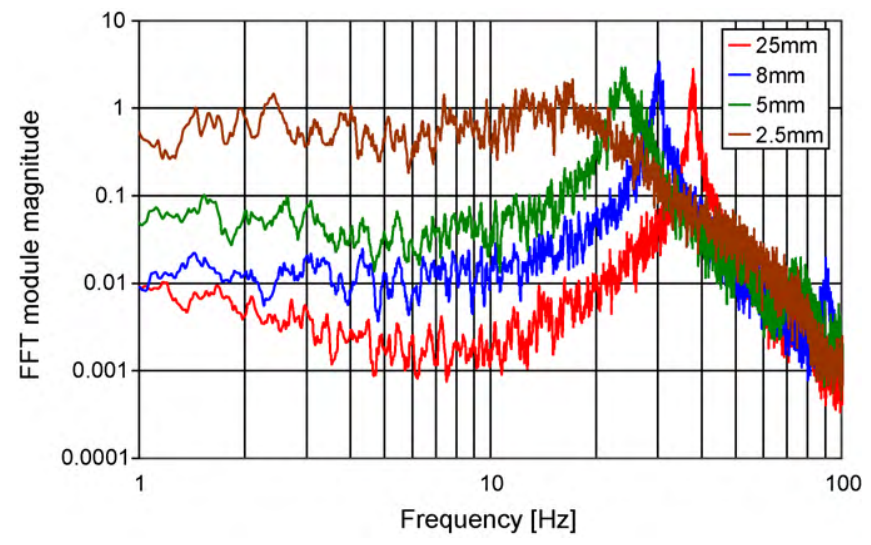

Fig. 13. Frequency amplitude spectra of the output voltage $V_{\mathrm{P}}$, measured for different values of the distance $d$, with an acceleration of the input vibrations $a_{\mathrm{rms}}=0.3 \mathrm{~g}$.

of the spectra similar to what predicted in the simulations of Fig. 6 . The cantilever bounces between the two stable states, evidencing as expected a higher voltage output over a wider frequency range with respect to the linear and quasi linear cases, especially at frequencies below resonance.

The voltage spectra of Fig. 13 indirectly provide information on the frequency distribution of the energy content potentially available at the converter output. Denoting with $S_{\mathrm{V}}(f)$ the power spectral density of the voltage $V_{\mathrm{P}}$, the power delivered to a resistive load $R_{\mathrm{L}}$ in the frequency range between $f_{1}$ and $f_{2}$ is given by:

$P=\int_{f_{1}}^{f_{2}} S_{\mathrm{V}}(f)\left|\frac{j 2 \pi f C_{\mathrm{P}} R_{\mathrm{L}}}{1+j 2 \pi f C_{\mathrm{P}} R_{\mathrm{L}}}\right|^{2} \frac{1}{R_{\mathrm{L}}} d f=\int_{f_{1}}^{f_{2}} S_{\mathrm{V}}(f)|H(f)|^{2} \frac{1}{R_{\mathrm{L}}} d f$

where the internal impedance of the converter has been assumed to be dominated by $C_{\mathrm{P}}$. The transfer function $H(f)$ is a weighting term that accounts for the adaptation over frequency between the source and load impedances. In the case where $f_{1} \gg 1 /\left(2 \pi C_{\mathrm{P}} R_{\mathrm{L}}\right)$, Eq. (9) simplifies to $P=\left(V_{\text {Prms }}\right)^{2} / R_{\mathrm{L}}$. This justifies why the rms output voltage over the considered bandwidth is a suitable 1 st order indicator of the power deliverable to the load in simplified conditions [30]. In any specific situations, Eq. (9) should be considered for the case at hand or its generalization where the appropriate load replaces the resistive load $R_{\mathrm{L}}$.

The locally linearized behaviour of the system when $d$ is varied is represented in Fig. 14, where the converter linearized resonance frequency is plotted versus the distance $d$, for a fixed amplitude of the mechanical excitation.

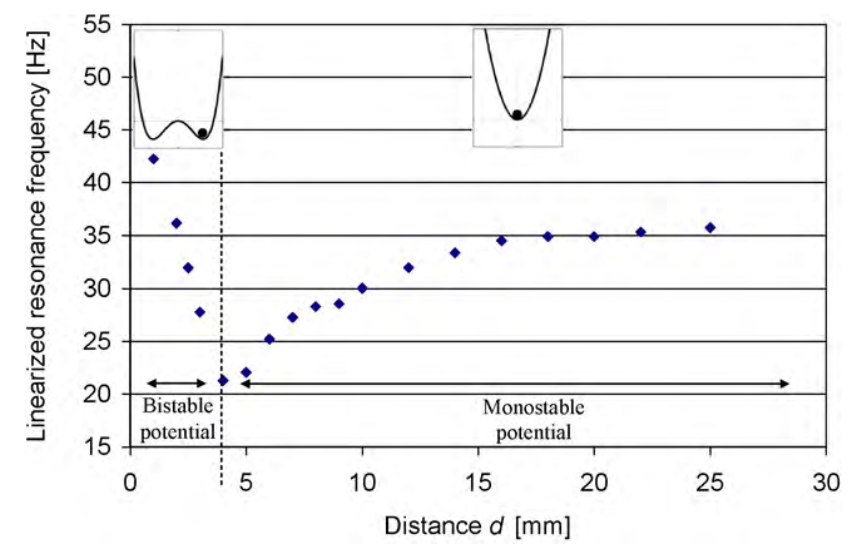

Fig. 14. Linearized resonance frequency of the converter for different values of the distance between the two magnets.
The excitation level was set low enough to avoid triggering bistability and jumps between the two stable states when the system potential energy becomes a double-well at low $d$. As a consequence, the system was kept oscillating around one of the equilibrium positions.

Despite of the residual dependence of the value of the linearized resonance frequency on the excitation amplitude, which would make the results of Fig. 14 quantitatively different by changing the input vibration level, the reported trend for a fixed excitation amplitude provides important information in accordance with the predictions by the simple model of Section 2 .

When the distance between the magnets is decreased starting from large values, as long as the system maintains a monostable potential energy, a decreasing trend of the resonance frequency of the linear or linearized system is visible due to a lowering of the equivalent stiffness. When $d$ decreases below $4 \mathrm{~mm}$, the potential energy of the system switches to the double-well shape. In this region, provided that the excitation is low enough as in this case, the system can sit in either equilibrium states and behaves as locally linear. The closer are the magnets, the higher is the confinement and the equivalent stiffness, which in turn causes the resonance frequency of the locally linearized system to increase with decreasing the distance $d$.

\section{Conclusions}

A nonlinear bistable piezoelectric converter for power harvesting from environmental vibrations was designed and tested. The bistability is obtained by properly exploiting a repulsive magnetic force. The basic theory behind this new approach was analytically described based on a simplified model and simulated by means of the MATLAB ${ }^{\circledR}$ SDE Toolbox. Analyses and simulations show that a nonlinear bistable converter gives better performances under wideband excitation with respect to a linear system, increasing the useful converter bandwidth and output level at parity of mechanical input. The converter was then realized by screen printing low-curing-temperature PZT films on steel cantilevers. A permanent magnet was fixed on the cantilever tip while an external permanent magnet, with opposite polarity, was placed in front of the cantilever tip on a micrometric stage. The converter was excited with band-limited white-noise mechanical vibrations and the amplitude and frequency spectrum of the open-circuit output voltage were measured at different values of the distance $d$ between the magnets. As expected, the experimental results initially show a decrease in the converter linearized resonance frequency and a corresponding increase in the displacement when the distance $d$ is decreased from large values as long as the system maintains a monostable quasi linear behaviour. When the distance is low enough, a bistable system is created, and bouncing between two stable states can occur. In this condition, the piezoelectric converter produces a marked improvement in the output voltage over a wider bandwidth with respect to the linear behaviour. An increase of up to $88 \%$ in the rms voltage was obtained in the tested conditions. This corresponds, in the simplified case of resistive load and frequency-independent transfer function between source and load impedances, to an expected increase of the power delivered to the load of about $250 \%$.

A proof of concept validation on a microscale device was also performed by using a MEMS U-shaped cantilever fabricated in SOI technology equipped with a miniaturized magnet. After this, the development is under way of MEMS bistable energy converters incorporating functional and structural materials such as piezoelectric and magnetic elements. 


\section{Acknowledgements}

The work was partially carried out under the project PRIN200720078ZCC92 co-funded by the Italian MIUR.

\section{References}

[1] K.A. Cook-Chennault, N. Thambi, A.M. Sastry, Powering MEMS portable devices-a review of non-regenerative and regenerative power supply systems with special emphasis on piezoelectric energy harvesting systems, Smart Mater. Struct. 17 (2008) 1-33.

[2] J.L. Gonzàlez, A. Rubio, F. Moll, Human powered piezoelectric batteries to supply power to wearable electronic devices, Int. J. Soc. Mater. Eng. Resour. 10 (2002) 33-40.

[3] C. Ó Mathúna, T. O’Donnell, R.V. Martinez-Catala, J. Rohan, B. O’Flynn, Energy scavenging for long-term deployable wireless sensor networks, Talanta 75 (2008) 613-623.

[4] R. Torah, P. Glynne-Jones, M. Tudor, T. O’Donnell, S. Roy, S. Beeby, Self-powered autonomous wireless sensor node using vibration energy harvesting, Meas. Sci. Technol. 19 (2008) 1-8.

[5] P.D. Mitcheson, E.M. Yeatman, G.K. Rao, A.S. Holmes, T.C. Green, Energy harvesting from human and machine motion for wireless electronic devices, Proc. IEEE 96 (2008) 1454-1486.

[6] T. Sterken, K. Baert, C. VanHoof, R. Puers, G. Borghs, P. Fiorini, Comparative modelling for vibration scavengers, in: Proceedings of IEEE Sensors 2004, vol. 3, Vienna, 24-27 October, 2004, pp. 1249-1252.

[7] E.P. James, M.J. Tudor, S.P. Beeby, N.R. Harris, P. Glynne-Jones, J.N. Ross, N.M. White, An investigation of self-powered systems for condition monitoring applications, Sens. Actuators 110 (2004) 171-176.

[8] S. Roundy, P.K. Wright, A piezoelectric vibration based generator for wireless electronics, Smart Mater. Struct. 13 (2004) 1131-1142.

[9] G.K. Ottman, H. Hofmann, A.C. Bhatt, G.A. Lesieutre, Adaptive piezoelectric energy harvesting circuit for wireless, remote power supply, IEEE Trans. Power Electronics 17 (2002) 669-676

[10] S.P. Beeby, M.J. Tudor, N.M. White, Energy harvesting vibration sources for microsystems applications, Meas. Sci. Technol. 17 (2006) 175-195.

[11] M. Ferrari, V. Ferrari, D. Marioli, A. Taroni, Modeling, fabrication and performance measurements of a piezoelectric energy converter for power harvesting in autonomous microsystems, IEEE Trans. Instrum. Meas. 55 (2006) 2096-2101.

[12] M. Ferrari, V. Ferrari, M. Guizzetti, D. Marioli, An autonomous battery-less sensor module powered by piezoelectric energy harvesting with RF transmission of multiple measurement signals, Smart Mater. Struct. 18 (2009) 1-9.

[13] D. Zhu, M.J. Tudor, S.P. Beeby, Strategies for increasing the operating frequency range of vibration energy harvesters: a review, Meas. Sci. Technol. 21 (2010) $1-29$.

[14] M. Ferrari, V. Ferrari, M. Guizzetti, D. Marioli, A. Taroni, Piezoelectric multifrequency energy converter for power harvesting in autonomous microsystems, Sens. Actuators 142 (2008) 329-335.

[15] I. Sari, T. Balkan, H. Kulah, An electromagnetic micro power generator for wideband environmental vibrations, Sens. Actuators 145-146 (2008) 405-413.

[16] B. Marinkovic, H. Koser, Smart Sand-a wide bandwidth vibration energy harvesting platform, Appl. Phys. Lett. 94 (2009) 103505.

[17] H. Xue, Y. Hu, Q.M. Wang, Broadband piezoelectric energy harvesting devices using multiple bimorphs with different operating frequencies, IEEE Trans. Ultrason. Ferr. 55 (2008) 2104-2108.

[18] B.P. Mann, N.D. Sims, Energy harvesting from the nonlinear oscillations of magnetic levitation, J. Sound Vib. 319 (2009) 515-530.

[19] S.M. Shahruz, Increasing the efficiency of energy scavengers by magnets, J. Comput. Nonlinear Dyn. 3 (2008)

[20] J.T. Lin, B. Lee, B. Alphenaar, The magnetic coupling of a piezoelectric cantilever for enhanced energy harvesting efficiency, Smart Mater. Struct. 19 (2010) 1-7.

[21] J. Qiu, J.H. Lang, A.H. Slocum, A curved-beam bistable mechanism, IEEE J. Microelectromech. Syst. 13 (2004) 137-146.

[22] R. Ramlan, M.J. Brennan, B.R. Mace, I. Kovacic, Potential benefits of a non-linear stiffness in an energy harvesting device, Nonlinear Dyn. 59 (2010) 545-558.

[23] S.C. Stanton, C.C. McGehee, B.P. Mann, Nonlinear dynamics for broadband energy harvesting: investigation of a bistable piezoelectric inertial generator, Phys. D 239 (2010) 640-653.

[24] F. Cottone, H. Vocca, L. Gammaitoni, Nonlinear energy harvesting, Phys. Rev. Lett. 102 (2009) 080601

[25] L. Gammaitoni, I. Neri, H. Vocca, Nonlinear oscillators for vibration energy harvesting, Appl. Phys. Lett. 94 (2009) 164102.

[26] P.E. Kloeden, E. Platen, Numerical solution of stochastic differential equations, Springer, 1992

[27] U. Picchini, SDE Toolbox: Simulation and Estimation of Stochastic Differential Equations with Matlab, http://sdetoolbox.sourceforge.net.
[28] M. Ferrari, V. Ferrari, M. Guizzetti, D. Marioli, Piezoelectric low-curingtemperature ink for sensors and power harvesting, in: Proceedings of the XIV Conference on Sensors and Microsystems, Pavia, Italy, 24-26 February, 2009, pp. 209-210.

[29] J.G. Webster, The Measurement, Instrumentation, and Sensors Handbook, CRC Press, 1999.

[30] E. Halvorsen, Energy harvesters driven by broadband random vibrations, IEEE J. Microelectromech. Syst. 17 (2008) 1061-1071

\section{Biographies}

Marco Ferrari was born in Brescia, Italy, in 1974. In 2002, he obtained the electronics engineering degree at the University of Brescia. In 2006 he received the research doctorate degree in electronic instrumentation at the same university. Since 2007 he has become an assistant professor at the Department of Electronics for Automation of the University of Brescia. His research activity deals with the energy conversion via the piezoelectric effect for powering autonomous microsystems and sensors for physical and chemical quantities with the related signal-conditioning electronics. In particular he is involved with piezoelectric acoustic-wave sensors in thick-film technology, design of oscillator circuits and frequency-output signal conditioners.

Vittorio Ferrari was born in Milan, Italy, in 1962. In 1988, he obtained the Laurea degree cum laude in physics at the University of Milan. In 1993 he received the Research Doctorate degree in electronic instrumentation at the University of Brescia. He has been an assistant professor and an associate professor at the Faculty of Engineering of the University of Brescia until 2001 and 2006, respectively. Since 2006 he has been a full professor of Electronics. His research activity is in the field of sensors and the related signal-conditioning electronics. Particular topics of interest are acoustic-wave piezoelectric sensors, microresonant sensors and MEMS, energy harvesting for autonomous sensors, oscillators for resonant sensors and frequency-output interface circuits. He is involved in national and international research programmes, and in projects in cooperation with industries.

Michele Guizzetti was born in Brescia, Italy, in 1981. In February 2006, he obtained the electronics engineering degree at the University of Brescia while in March 2010 he received the Research Doctorate degree in Electronic Instrumentation at the same university. Since March 2010 he is collaborating with the Department of Information Engineering of the University of Brescia. His research activity deals with power harvesting and autonomous systems. In particular he is involved with piezoelectric and thermoelectric energy conversion.

Bruno Andò received the M.S. in Electronic Engineering and the Ph.D. in Electrical Engineering from the Università di Catania, Italy, in 1994 and 1999, respectively. From 1999 to 2001, he worked as a researcher with the Electrical and Electronic Measurement Group of the University of Catania - DEES. In 2002, he became an assistant professor with the same staff. His main research interests are sensors design and optimization including advanced multi-sensors architecture for visually impaired people, characterization of new materials for sensors, nonlinear techniques for signal processing with particular interest in stochastic resonance and dithering applications; characterization and conditioning; distributed measurement systems. During his activity Dr. Andò has co-authored several scientific papers, presented in international conferences and published in international journals and books.

Salvatore Baglio received the "Laurea" and Ph.D. degrees from the University of Catania, Catania, Italy, in 1990 and 1994, respectively. Since 1996 he is with the Dipartimento di Ingeoneria Elettrica Elettronica e dei Sistemi, University of Catania where he is currently Associate Professor. Prof. Baglio teaches courses in "measurement theory", "electronic instrumentations", and "integrated microsensors". He is a co-author of more than 250 scientific publications, which include books, chapters in books, papers in international journals and proceedings of international conferences. He is also co-holder of several U.S. patents. Prof. Baglio is Senior Member of the IEEE, he has served as Associate Editor for the IEEE Transactions on Circuits and Systems-I, as Distinguished Lecturer for the IEEE Circuits and Systems Society and is currently Associate Editor of the IEEE Transaction on Instrumentation and Measurements. His research interests are mainly focused on microsensors and nanosensors, hysteretic materials for sensors, nonlinear dynamics for transducers.

Carlo Trigona was born in Siracusa, Italy, in 1981. He received his M.S. degree in Automation Engineering and Control of Complex System and the Ph.D. degree in Electronic, Automation and Control of Complex System from University of Catania, Catania, Italy in 2006 and 2009, respectively. He is a co-author of more than 40 scientific publications, which include chapters in books, papers in international journals and proceedings of international conferences. His research interests include microsystems and microsensors, fluxgate magnetometers, analog and digital electronic circuit designs. 